\title{
Predictive Accuracy of Urinary neutrophil gelatinase associated lipocalin (NGAL) for renal parenchymal involvement in Children with Acute Pyelonephritis
}

\author{
Kambiz Ghasemi ${ }^{1}$, Maryam Esteghamati ${ }^{1}$, Sara Borzoo ${ }^{2}$, Erfan Parvaneh ${ }^{3}$, Samira Borzoo ${ }^{4}$
}

${ }^{1}$ M.D., Assistant Professor, Department of Pediatric Nephrology, Faculty of Medicine, Hormozgan University of Medical Sciences, Bandar Abbas, Iran

${ }^{2}$ M.D., Pediatric Resident, Department of Pediatrics, Faculty of Medicine, Hormozgan University of Medical Sciences, Bandar Abbas, Iran

${ }^{3}$ Medical Student, Department of Pediatrics, Faculty of Medicine, Isfahan University of Medical Sciences, Isfahan, Iran

${ }^{4}$ M.Sc., Rehabilitation Administration, MPH Student of Health Policy Research Center, Shiraz University of Medical Sciences, Shiraz, Iran

Type of article: Original

\begin{abstract}
Introduction: Urinary tract infections (UTIs) are among the most prevalent infections in children and infants. Early and accurate detection of renal parenchymal involvement in UTI is necessary for decision making and determining treatment strategies. The aim of this study was to determine the predictive accuracy of urinary neutrophil gelatinase-associated lipocalin (NGAL) for renal parenchymal involvement in children with acute pyelonephritis.

Methods: This descriptive, cross-sectional study was conducted in 2014 on children who had been diagnosed with UTI. Children who were admitted to Koodakan Hospital in Bandar Abbas, Hormozgan Province, Iran, and whose ages ranged from two months to 14 years were enrolled in the study. Urine samples were taken to conduct urinary NGAL tests, urine cultures, and urinalyses. In addition, some blood samples were collected for the purpose of determining leukocyte count and C-reactive protein (CRP) and to conduct erythrocyte sedimentation rate (ESR) tests. All patients underwent a dimercaptosuccinic acid (DMSA) scan. SPSS software was used to analyze the data.

Results: Among the participants in the study, 29 were male (32\%), and 60 were female (68\%). The mean age of the children who participated in the study was $2.99 \pm 2.94$ years. The results of the Kruskal-Wallis test showed a significant increase in the urinary NGAL level, an increase in the CRP level, and higher DMSA scan grades ( $<<$ 0.001 ). The cutoff point amounted to $>5 \mathrm{mg} / \mathrm{l}$, having the negative predictive value (NPV) of $76.3 \%$, the specificity of $97.83 \%$, the positive predictive value (PPV) of $96.7 \%$, and the sensitivity of $67.4 \%$.

Conclusion: Urinary NGAL is not sensitive enough for the prediction of renal parenchymal involvement, but it is a specific marker.
\end{abstract}

Keywords: renal parenchymal involvement, C-reactive protein, urinary tract infections, urinary NGAL

\section{Introduction}

For infants and children, urinary tract infections (UTIs) are regarded as one of the most prevalent infections (1). UTIs are prevalent in children, afflicting $3.3-5.3 \%$ of infants who have a fever $(2,3)$. UTIs are particularly prevalent in Caucasian girls, and they are usually not accompanied by a fever (3). The non-particular essence of the symptoms among infants and young children who have a fever produces problems in clinically distinguishing upper UTIs with renal parenchymal involvement from lower UTIs with no renal parenchymal involvement (2-4). Notwithstanding the fact that upper UTIs are often referred to as acute pyelonephritis, we chose not to use that term because it is a

\section{Corresponding author:}

Dr. Sara Borzoo. M.D., Department of Pediatrics, Faculty of Medicine, Hormozgan University of Medical Sciences Tel: +98.9177314740, E-mail: Saraborzoo@yahoo.com

Received: June 25, 2015, Accepted: September 04, 2015, Published: February 2016

iThenticate screening: August 17, 2015, English editing: November 01, 2015, Quality control: January 10, 2016

(C) 2016 The Authors. This is an open access article under the terms of the Creative Commons Attribution-NonCommercialNoDerivs License, which permits use and distribution in any medium, provided the original work is properly cited, the use is non-commercial and no modifications or adaptations are made. 
clinical diagnosis that should not be used for infants or young children. Due to its connection with renal scarring, the exact recognition and timely treatment of upper UTI are important $(5,6)$. During the last years of life, one of the most intricate problems of UTI is the kidney injury that results in hypertension and, finally, end-stage renal disease $(7,8)$. Hence, a timely and well-arranged diagnostic test can be useful for the early recognition of renal parenchymal involvement that needs more efficient treatment than lower UTI, as well as more careful observation by the patient. Technetium Tc 99m dimercaptosuccinic acid (99mTc-DMSA) renal scintigraphy commonly is used to determine the severity of the injury to the kidney and the resulting scarring in patients afflicted by acute pyelonephritis (9). DMSA is regarded as the determining factor regarding the diagnosis of renal parenchymal involvement and the evaluation of the severity and advancement of the renal parenchymal damage. Some laboratory indicators have been used to predict the extent of injury to the kidney and the extent of scarring. Among these indicators, erythrocyte sedimentation rate (ESR) and leukocyte count are the most frequently used in predicting the results of the DMSA scan (10-12).

Patients who have higher leukocyte counts and ESR are at greater risk of parenchymal involvement. C-reactive protein (CRP) is another indicator that commonly is used in qualitative assessments. The progress of renal scarring poses higher risks to the patients who have positive CRP rates. None of the indicators mentioned to this point has the necessary precision to predict renal injury. Hence, researchers make use of novel indicators that can be of higher diagnostic value to foresee renal injuries (13-15); such indicators include the proteins that determine the rate of urinary neutrophil gelatinase associated with lipocalin (NGAL). NGAL is a novel protein detected in neutrophil granules, and is one of the constituents of the innate immune system (16-18). This is likewise demonstrated by some other cells, such as renal tubular and epithelial cells, as well as in epithelial cell damage of the liver, kidneys, lungs, and colon (19). This indicator makes it possible to obtain a quick and certain detection of urinary tract infection. Its sensitivity ranges from 91 to $98 \%$, and the specificity of this method has been reported to be $76-95 \%$ in various studies. In several studies this biomarker has been assessed as a quick and non-invasive test (20). In 2009, Yilmaz et al. conducted research to determine the level of urinary NGAL, and they stated that it may be regarded as a novel and valid indicator of UTI. They concluded that urinary NGAL levels could be used to diagnose UTI because its levels in a UTI group were conspicuously higher than in the control group. The test's urinary NGAL sensitivity is 97\% (21). Won Hee Seo et al. conducted the research in 2014 in China. The NGAL levels were significantly higher in cortical defects UTI than in the control group with no cortical defects (22). The aim of this study was to determine the diagnostic accuracy of the urinary NGAL test in the detection of renal parenchymal involvement in children with acute pyelonephritis.

\section{Material and Methods}

\subsection{Study setting and design}

This descriptive cross-sectional study was done in 2014 on children who had been diagnosed with UTI. Children between the ages of 2 months and 14 years old who were admitted to Koodakan Hospital in Bandar Abbas in Hormozgan Province, Iran, were enrolled in the study.

\subsection{Sampling}

Eighty-nine children with fever $\left(38.5{ }^{\circ} \mathrm{C}\right.$ or higher) and previous UTI $\left(>10^{5}\right.$ colony count per ml culture in midstream clean voiding sample and more than $10^{3}$ colony count in sampling by catheter) were enrolled in the study. Children with anomalies of the urinary system, neurogenic bladder, and who had previous surgery in the urinary tract system were excluded from the study. Urine analysis, urine culture, urinary NGAL level, CRP level, ESR, and complete blood count tests were conducted for all of the children in the study.

\subsection{Dimercaptosuccinic Acid Renal Scintigraphy}

All DMSA scans were performed in a single center under the supervision of a nuclear medicine specialist using a Siemens E CAM 2009 device. In order to perform imaging, technetium was injected intravenously. The following grading was used for interpretation of scan results:

1) Grade 0: Normal DMSA scan

2) Grade I: <2 renal contour defects

3) Grade II: 2 renal contour defects with areas of normal parenchyma

4) Grade III: Diffuse reduction in uptake 


\subsection{Urinary NGAL Measurement}

To measure the NGAL concentration in the urine, 15 to $20 \mathrm{ml}$ of each patient's urine were used. The urine samples were kept at 2 to $8^{\circ} \mathrm{C}$. The ELISA method was used to measure the NGAL concentrations in the urine, and the results were reported in $\mathrm{ng} / \mathrm{ml}$.

\subsection{Other Laboratory Tests}

A Sysmex KX-21N device (Japan) was used to perform the complete blood count test. Also, a Lendo device (Spain) was used to measure ESR. We measured the CRP concentration manually.

\subsection{Research ethics}

This research was approved by Ethics Committee at Hormozgan University of Medical Sciences. The patients were free to discontinue their participation in the study at any time. The patients' information and laboratory test results were confidential.

\subsection{Statistical Analysis}

We used version 20.0 of SPSS IBM, Inc. software and MedCalc software to analyze the data. For the analyses of the data, descriptive statistics were used, including mean, standard deviation, frequency, and percentage. Also, the Kruskal-Wallis test was used to compare urinary NGAL concentrations based on the DMSA scan and CRP levels. We calculated specificity, sensitivity, positive predictive value (PPV), and negative predictive value (NPV) for urinary NGAL using ROC curve analysis. A p value $<0.05$ was assumed to be significant.

\section{Results}

\subsection{Sociodemographic and general findings}

Twenty-nine of the participants were male (32\%), and 60 were female $(68 \%)$. The mean age of the children in the study was $2.99 \pm 2.94$ years. The average duration of hospitalization was 3.91 days in our study. Abnormal urine analyses were reported in 41 patients (46.06\%). Also CRP was negative in 31 patients $(34.8 \%)$ and was trace positive in 3 patients (3.4\%). Also $1+, 2+$, and $3+$ CRP tests were reported in 17 patients (19.1\%), 18 patients $(20.2 \%)$, and 20 patients $(22.5 \%)$, respectively. DMSA scan was normal in 46 patients $(51.7 \%)$, grade I in 23 patients $(25.8 \%)$, and grades II and III in 20 patients $(22.4 \%)$.

\subsection{Urinary NGAL concentration and DMSA scan}

The results of the Kruskal-Wallis test showed that there was a significant difference in the concentrations of NGAL in the children's urine samples based on the results of the DMSA scan $(p<0.001)$ (Table 1).

Table 1. Urinary NGAL level according to dimercaptosuccinic acid scintigraphy

\begin{tabular}{|l|l|l|l|}
\hline DMSA & $\mathrm{n}$ & Mean \pm Standard deviation & $\mathrm{p}$-value \\
\hline Normal & 46 & $1.66 \pm 1.78$ & $<0.001$ \\
grade I & 23 & $6.33 \pm 4.16$ & \\
\cline { 1 - 2 } grade II & 14 & $8.92 \pm 5.10$ & \\
\hline grade III & 6 & $14.88 \pm 3.82$ & \\
\hline
\end{tabular}

\subsection{Urinary $N G A L$ concentration and $C R P$}

Based on results of the Kruskal-Wallis test, the NGAL concentrations in the children's urine samples were significantly higher in the children who had higher CRP levels $(\mathrm{p}<0.001)$ (Table 2).

Table 2. Urinary NGAL level according to C-reactive protein (CRP) Level

\begin{tabular}{|l|l|l|l|}
\hline CRP & $\mathrm{n}$ & Mean \pm Standard deviation & $\mathrm{p}$ value \\
\hline Negative & 31 & $2.41 \pm 4.63$ & \multirow{2}{*}{$<0.001$} \\
\cline { 1 - 2 } & 17 & $3.97 \pm 4.06$ & \\
\cline { 1 - 2 } & 18 & $8.24 \pm 4.12$ & \\
\cline { 1 - 2 }+ & 20 & $6.63 \pm 5.57$ & \\
\hline Trace & 3 & $4.30 \pm 4.09$ & \\
\hline
\end{tabular}




\subsection{Sensitivity, specificity, PPV, and NPV of urinary NGAL, leukocyte count, and ESR}

The results the analyses of the ROC curves were used to determine the accuracy of using the NGAL concentration in the children's urine in predicting the DMSA scan Figure 1 shows the comparison of the ROC curve for the accuracy of NGAL in the urine, leukocyte count, and ESR in prediction of the DMSA scan in children with pyelonephritis. A cutoff point for NGAL concentration in the urine was selected as $>5 \mathrm{mg} / \mathrm{l}$ for prediction of positive results in the DMSA scan. For the NGAL concentration in the urine, the area under the curve (AUC) was 0.902 (95\% CI: 0.820 0.955) $(\mathrm{p}<0.001)$. The NGAL concentration in the urine had a sensitivity of $67.4 \%(95 \%$ CI: $51.5-80.9)$ and a specificity of $97.83 \%$ (95\% CI: 88.5-99.9), a PPV of 96.7\% (95\% CI: 82.8-99.9) and an NPV of 76.3\% (95\% CI: 63.4-86.4) for prediction of the results of the DMSA scan. The AUC was 0.829 (95\% CI: 0.735-0.901) for leukocyte count $(\mathrm{p}<0.001)$. A leukocyte count $>11800 \times 1012 / \mathrm{L}$ was selected as the cutoff point for prediction of a positive DMSA scan test with a sensitivity of $76.74 \%$ (95\% CI: $61.4-88.2)$ and a specificity of $80.43 \%$ (95\% CI: 66.1-90.6), a PPV of 78.6\% (95\% CI: 63.12-89.7), and an NPV of 78.7\% (95\% CI: $64.2-81.0)$. For ESR, the AUC was 0.849 $(95 \%$ CI: $0.758-0.916)(\mathrm{p}<0.001)$. A cutoff point of $>32 \mathrm{~mm} / \mathrm{h}$ was selected for ESR for the prediction of a positive DMSA scan with a sensitivity of 76.74\% (95\% CI: $61.4-88.2)$, a specificity of $86.96 \%$ (95\% CI: 73.7-95.1), a PPV of $86.4 \%$ (95\% CI: 69.5-94.1), and an NPV of 80.0\% (95\% CI: 66.3-90.1) (Table 3).

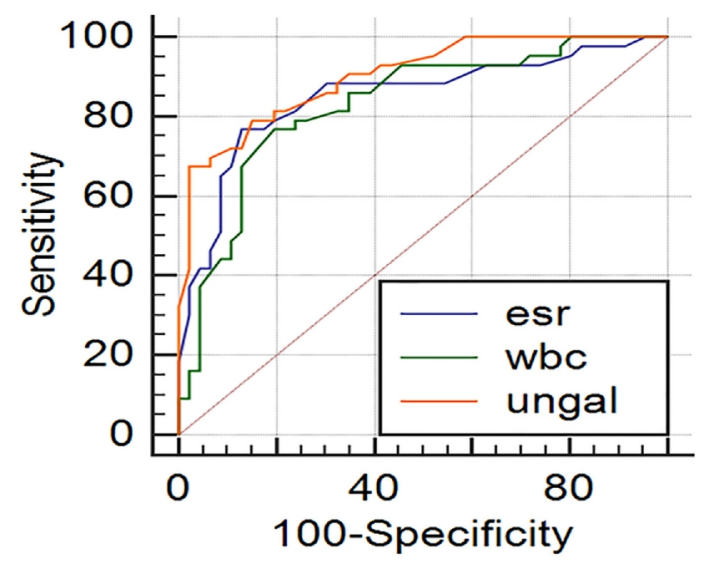

Figure 1. Receiver operating characteristic curves for urinary NGAL, leukocyte count, and erythrocyte sedimentation rate for prediction of dimercaptosuccinic acid scintigraphy results

Table 3. Diagnostic accuracy of urinary NGAL, leukocyte count, and erythrocyte sedimentation rate for prediction of dimercaptosuccinic acid scintigraphy results

\begin{tabular}{|l|l|l|l|l|}
\hline Diagnosis Test & Sensitivity & Specificity & $\mathrm{PPV}^{1}$ & $\mathrm{NPV}^{2}$ \\
\hline Urinary NGAL & $67.44 \%$ & $97.83 \%$ & $96.7 \%$ & $76.3 \%$ \\
\hline Leukocyte count & $76.7 \%$ & $80.4 \%$ & $78.6 \%$ & $78.7 \%$ \\
\hline Erythrocyte sedimentation rate & $76.7 \%$ & $87 \%$ & $84.6 \%$ & $80 \%$ \\
\hline
\end{tabular}

1: Positive Predictive Value, Negative Predictive Value

\section{Discussion}

Especially in asymptomatic infants, the timely recognition of upper UTIs is a difficult task (2-4). However, differentiating between upper and lower UTI is essential because renal parenchymal involvement can produce perpetual renal scarring that can result into chronic renal failure and hypertension (5-6). As a result, recognizing renal parenchymal involvement and treating it in a timely manner are very important. Injuries and renal scarring are among the most significant effects of an undiagnosed and untreated UTI. Thus, it is very important to diagnose and treat this condition as early as possible. The DMSA scan is regarded as a good way of detecting kidney injuries and renal scarring (23). During the critical stage of the infection, performing a DMSA scan is of high importance in evaluating renal parenchymal involvement, with an approximate sensitivity of $93 \%$ (10). Nevertheless, there are some factors that restrict the prevalent utilization of DMSA scintigraphy. These factors include the related costs, patients being exposed to radiation, restricted accessibility, and the impossibility of distinguishing between critical renal parenchymal involvement and old scarring without conducting additional DMSA scans in the future. 
Renal scan results have demonstrated that the excretion of NGAL in the urine increases after renal damage, and they also may be used for the timely recognition of critical pyelonephritis (21). Some researchers have demonstrated that the determination of the concentration of NGAL in the urine is the most trustworthy, non-invasive way of diagnosing of acute pyelonephritis (24). A study conducted by Won Hee Seo demonstrated that the median concentration of NGAL in the urine in the UTI group with cortical defect was significantly higher than the concentration in the UTI group without cortical defect, i.e., $106.5 \mathrm{vs.} 60 \mathrm{ng} / \mathrm{ml}$ ) (22). Our study showed the accuracy of the NGAL concentration for predicting renal parenchymal involvement in children with acute pyelonephritis. In our study, a cutoff concentration of $61 \mathrm{ng} / \mathrm{ml}$ of NGAL in urine was reported for detecting pyelonephritis with a sensitivity of $75 \%$ and a specificity of $78.3 \%$. Yilmaz reported that the mean urinary NGAL level was significantly higher in the group with UTI than in the control group, i.e., $91.02 \mathrm{vs.} 14.29 \mathrm{ng} / \mathrm{ml}$, and the cutoff was $20 \mathrm{ng} / \mathrm{ml}$ for urinary NGAL for diagnosis of UTI with a sensitivity of $97 \%$ and a specificity of $96 \%$ (21). In this study, the cutoff point was $>5 \mathrm{mg} / \mathrm{l}$, having the NPV of $76.3 \%$, the specificity of $97.83 \%$, the PPV of $96.7 \%$, and the sensitivity of $67.4 \%$. Based on our results, leukocyte count and ESR have the same sensitivity and are higher in comparison to urinary NGAL. Urinary NGAL is more specific for diagnosing renal parenchymal involvement. Based on the results of our study, the PPV is higher in ESR and CRP than in urinary NGAL; also, the NPV for leukocyte count was higher than urinary NGAL. In our results, major differences were identified in the urinary NGAL concentration among patients based on the CRP level. This finding showed that urinary NGAL is a more dependable biological marker of higher specificity than leukocyte count and ESR.

Our results indicated that urinary NGAL should not be used to predict renal parenchymal involvement due to its high costs and its unavailability to all health clinics and patients. Furthermore, making use of other tests, such as leukocyte count and ESR, would provide a more accurate prediction. A probable explanation of these results may be that the excretion of urinary NGAL occurs with renal parenchymal injury because the severity of glomerular filtration is lessened extensively. The results of the urinary NGAL test can be normal in the individuals who are in the earlier phases of renal injury or who have a mild renal injury (25). Also, we used CRP in our research. Contrary to some researchers who utilized quantitative CRP measures, we used qualitative measures because the qualitative method was available and was the prevalent method in our hospital. It also was demonstrated that CRP level is linked to DMSA scan results and that an increase in the CRP level is associated with greater risks of an abnormal DMSA scan. Hence, as another detection indictor, CRP can be used to predict the results of DMSA scans.

\section{Conclusions}

The results of this research showed that, while urinary NGAL is specific enough to be used as a diagnostic marker for the prediction of renal parenchymal involvement, it is not sensitive enough to be used for this purpose. The clinical application of these results is to make better diagnostic strategies for the detection of renal parenchymal involvement in UTI in children. We recommend using other common markers, such as ESR, leukocyte count, and CRP in combination with urinary NGAL to predictrenal parenchymal involvement in children with UTI. Future studies should focus on combinations of these tests for early and accurate detection of renal parenchymal involvement.

\section{Acknowledgments:}

This paper is extracted from thesis provided by Sara Borzoo for fulfilment of requirements required for degree of Medical Doctorate in pediatric diseases. The authors gratefully acknowledge the financial support for this work that was provided by clinical research development center of Koodakan hospital in Hormozgan University of Medical Sciences.

\section{Conflict of Interest:}

There is no conflict of interest to be declared.

\section{Authors' contributions:}

All authors contributed to this project and article equally. All authors read and approved the final manuscript.

\section{References}

1) Wald E. Urinary tract infections in infants and children: a comprehensive overview. Curr Opin Pediatr. 2004;16(1):85-8. PMID: 14758120

2) Hoberman A, Chao HP, Keller DM, Hickey R, Davis HW, Ellis D. Prevalence of urinary tract infection in febrile infants. J Pediatr. 1993;123(1):17-23. doi: 10.1016/S0022-3476(05)81531-8. PMID: 8320616 
3) Shaw KN, Gorelick M, McGowan KL, Yakscoe NM, Schwartz JS. Prevalence of urinary tract infection in febrile young children in the emergency department. Pediatrics. 1998;102(2):e16. doi: 10.1542/peds.102.2.e16. PMID: 9685461

4) Hoberman A, Wald ER. Urinary tract infections in young febrile children. Pediatr Infect Dis J. 1997;16(1):11-7. doi: 10.1097/00006454-199701000-00004. PMID: 9002094

5) Lin KY, Chiu NT, Chen MJ, Lai CH, Huang JJ, Wang YT, et al. Acute pyelonephritis and sequelae of renal scar in pediatric first febrile urinary tract infection. Pediatr Nephrol. 2003;18(4):362-5. doi: 10.1007/s00467-003-1109-1. PMID: 12700963

6) Jakobsson B, Berg U, Svensson L. Renal scarring after acute pyelonephritis. Arch Dis Child. 1994;70(2):111-5. PMID: 8129430, PMCID: PMC1029711

7) Martinell J, Lidin-Janson G, Jagenburg R, Sivertsson R, Claesson I, Jodal U. Girls prone to urinary infections followed into adulthood. Indices of renal disease. Pediatr Nephrol. 1996;10(2):139-42. PMID: 8703696

8) Jacobson SH, Eklof O, Eriksson CG, Lins LE, Tidgren B, Winberg J. Development of hypertension and uraemia after pyelonephritis in childhood: 27 year follow up. BMJ. 1989;299(6701):703-6. PMID: 2508881, PMCID: PMC1837515

9) Dick PT, Feldman W. Routine diagnostic imaging for childhood urinary tract infections: a systematic overview. J Pediatr. 1996;128(1):15-22. PMID: 8551409

10) Lavocat MP, Granjon D, Allard D, Gay C, Freycon MT, Dubois F. Imaging of pyelonephritis. Pediatr Radiol. 1997;27(2):159-65. doi: 10.1007/s002470050091. PMID: 9028852

11) Falakaflaki B, Mazloomzadeh S, Molaei Z. The Incidence of Renal Scarring and its Related Factors in Children with First Pyelonephritis. Journal of Pediatric Nephrology. 2014;2(1):30-4.

12) Jaksic E, Bogdanovic R, Artiko V, Saranovic DS, Petrasinovic Z, Petrovic M, et al. Diagnostic role of initial renal cortical scintigraphy in children with the first episode of acute pyelonephritis. Ann Nucl Med. 2011;25(1):37-43. doi: 10.1007/s12149-010-0431-5. PMID: 21080122

13) Mantadakis E, Plessa E, Vouloumanou EK, Karageorgopoulos DE, Chatzimichael A, Falagas ME. Serum procalcitonin for prediction of renal parenchymal involvement in children with urinary tract infections: a meta-analysis of prospective clinical studies. J Pediatr. 2009;155(6):875-81. doi: 10.1016/j.jpeds.2009.06.037. PMID: 19850301

14) Sheu JN, Chang HM, Chen SM, Hung TW, Lue KH. The role of procalcitonin for acute pyelonephritis and subsequent renal scarring in infants and young children. J Urol. 2011;186(5):2002-8. doi: 10.1016/j.juro.2011.07.025. PMID: 21944121

15) Leroy S, Gervaix A. Procalcitonin: a key marker in children with urinary tract infection. Adv Urol. 2011;2011:397618. doi: 10.1155/2011/397618. PMID: 21274426, PMCID: PMC3026964

16) Xu SY, Carlson M, Engstrom A, Garcia R, Peterson CG, Venge P. Purification and characterization of a human neutrophil lipocalin (HNL) from the secondary granules of human neutrophils. Scand J Clin Lab Invest. 1994;54(5):365-76. PMID: 7997842

17) Flo TH, Smith KD, Sato S, Rodriguez DJ, Holmes MA, Strong RK, et al. Lipocalin 2 mediates an innate immune response to bacterial infection by sequestrating iron. Nature. 2004;432(7019):917-21. doi: 10.1038/nature03104. PMID: 15531878

18) Berger T, Togawa A, Duncan GS, Elia AJ, You-Ten A, Wakeham A, et al. Lipocalin 2-deficient mice exhibit increased sensitivity to Escherichia coli infection but not to ischemia-reperfusion injury. Proc Natl Acad Sci U S A. 2006;103(6):1834-9. doi: 10.1073/pnas.0510847103. PMID: 16446425, PMCID: PMC1413671

19) Nguyen MT, Devarajan P. Biomarkers for the early detection of acute kidney injury. Pediatr Nephrol. 2008;23(12):2151-7. doi: 10.1007/s00467-007-0470-x. PMID: 17394022

20) Makris K, Markou N, Evodia E, Dimopoulou E, Drakopoulos I, Ntetsika K, et al. Urinary neutrophil gelatinase-associated lipocalin (NGAL) as an early marker of acute kidney injury in critically ill multiple trauma patients. Clin Chem Lab Med. 2009;47(1):79-82. doi: 10.1515/CCLM.2009.004. PMID: 19055468

21) Yilmaz A, Sevketoglu E, Gedikbasi A, Karyagar S, Kiyak A, Mulazimoglu M, et al. Early prediction of urinary tract infection with urinary neutrophil gelatinase associated lipocalin. Pediatr Nephrol. 2009;24(12):2387-92. doi: 10.1007/s00467-009-1279-6. PMID: 19649660

22) Seo WH, Nam SW, Lee EH, Je BK, Yim HE, Choi BM. A rapid plasma neutrophil gelatinase-associated lipocalin assay for diagnosis of acute pyelonephritis in infants with acute febrile urinary tract infections: a preliminary study. Eur J Pediatr. 2014;173(2):229-32. doi: 10.1007/s00431-013-2112-6. PMID: 23918295 
23) Mohkam M, Maham S, Rahmani A, Naghi I, Otokesh B, Raiiati H, et al. Technetium Tc 99m dimercaptosuccinic acid renal scintigraphy in children with acute pyelonephritis: correlation with other imaging tests. Iran J Kidney Dis. 2010;4(4):297-301. PMID: 20852370

24) Oh HS, Bae SP, Kim SS, Park KB. Usefulness of Spot Urine beta2-microglobulin in Pediatric Acute Pyelonephritis. Soonchunhyang Med Sci. 2012;18:91-4.

25) Jacobson SH, Lindvall N, Lins L-E. Renal Size, Glomerular Function and Urinary Excretion of Albumin and $\beta 2$-Microglobulin in Patients with Renal Scarring Due to Pyelonephritis. Acta Medica Scandinavica. 1987;222(3):261-6. doi: 10.1111/j.0954-6820.1987.tb10668.x. 